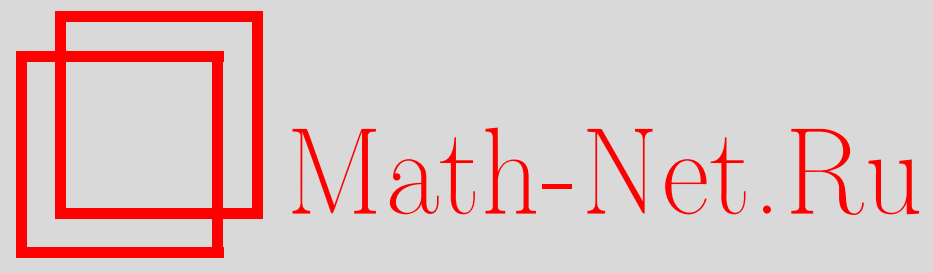

Д. Златопольский, Бывают ли отрицательные цифры?, Квант, 2021, номер 4, 11-16

DOI: https://doi.org/10.4213/kvant20210402

Использование Общероссийского математического портала Math-Net.Ru подразумевает, что вы прочитали и согласны с пользовательским соглашением http://www . mathnet.ru/rus/agreement

Параметры загрузки:

IP: 3.89.197.203

26 апреля 2023 г., 16:56:38 


\section{Бывают ли \\ отрицательные цифры?}

\section{Д.ЗЛАТОПОЛЬСКИЙ}

\section{Что такое отрицательные цифры}

Как ни странно, ответ на вопрос, вынесенный в название статьи: «Да, бывают!» Впервые отрицательные цифры предложил использовать еще в 1726 году английский математик Джон Колсон в своей статье «Краткое изложение отрицательноположительной арифметики» [1]. Чтобы отличить отрицательные цифры от «обычных», он предложил обозначать их с черточкой над цифрой. Так, в начале статьи ученый приводит число $3 \overline{7} 09 \overline{2} \overline{8} 65 \overline{7} 3 \overline{9} \overline{6} \overline{1} 47 \overline{2}$ и далее показывает, что оно соответствует «обычному» числу 2308726432039468, хотя приведенный пример не совсем соответствует цели, которую, как пишет сам Колсон, он ставит, вводя отрицательные цифры. А цель эта такая - упрощение арифметических операций над большими числами путем отказа от использования «больших» цифр $6,7,8$ и 9 и замены их значениями с отрицательными цифрами $1 \overline{4}, 1 \overline{3}, 1 \overline{2}$ и $1 \overline{1}$. Здесь $1 \overline{4}=10-4$, так же, как $14=10+4$ (аналогично для $1 \overline{3}, 1 \overline{2}, 1 \overline{1}$ ). В дальнейшем Колсон приводит пример такого преобразования - вместо числа 181937462 использовать при расчетах число $2 \overline{2} 2 \overline{1} 4 \overline{3} 5 \overline{4} 2 .{ }^{1}$

\section{Удобнее складывать}

Показывая преимущества (по его мнению) своей системы, Колсон приводит такой пример сложения нескольких чисел:

\footnotetext{
1 Конечно, Колсон описывает в статье правила преобразования чисел с цифрами $6,7,8$ и 9 в числа с отрицательными цифрами и обратно.
}

$$
\begin{array}{r}
2 \overline{1} 5 \overline{3} \overline{1} 40 \overline{3} 3 \overline{1} \overline{2} \overline{1} 3 \\
50 \overline{4} \overline{2} 03 \overline{1} \overline{4} 55 \overline{1} \overline{2} \\
4 \overline{3} 10 \overline{2} \overline{3} 10 \overline{2} \overline{4} \overline{1} \overline{3} \\
5 \overline{1} 3 \overline{4} \overline{2} 1 \overline{1} 03 \overline{2} 1 \\
2 \overline{1} 30 \overline{4} 2 \overline{1} 03 \overline{2} \\
1 \overline{3} 2021 \overline{2} 2 \overline{4} \\
13 \overline{2} \overline{2} 43 \overline{1} 5 \\
\hline \overline{3} 33 \overline{3} 214 \overline{1} \overline{3} \overline{4} 3 \overline{1} \overline{2}
\end{array}
$$

Видно, что если бы слагаемые были представлены в обычной десятичной записи, то пришлось бы, например, в последнем разряде складывать цифры

$$
\begin{aligned}
& 3 \\
& 8 \\
& 7 \\
& 1 \\
& 8 \\
& 6 \\
& 5
\end{aligned}
$$

в то время как в приведенном варианте нужно сложить цифры

$$
\begin{aligned}
& \frac{3}{2} \\
& \overline{3} \\
& 1 \\
& \overline{2} \\
& \overline{4} \\
& 5
\end{aligned}
$$

что, конечно, проще (при этом уменьшается и вероятность ошибки).

DOI: https://doi.org/10.4213/kvant20210402 


\section{Удобнее умножать}

Главным преимуществом своей системы Колсон считал упрощение умножения чисел в ней. В его системе таблица умножения включает только 25 небольших значений (каждое из которых может быть со знаками «+» и «-»):

\begin{tabular}{|c|c|c|c|c|c|c|c|c|c|c|}
\hline$\times$ & 1 & -1 & 2 & -2 & 3 & -3 & 4 & -4 & 5 & -5 \\
\hline 1 & 1 & -1 & 2 & -2 & 3 & -3 & 4 & -4 & 5 & -5 \\
\hline-1 & -1 & 1 & -2 & 2 & -3 & 3 & -4 & 4 & -5 & 5 \\
\hline 2 & 2 & -2 & 4 & -4 & 6 & -6 & 8 & -8 & 10 & -10 \\
\hline-2 & -2 & 2 & -4 & 4 & -6 & 6 & -8 & 8 & -10 & 10 \\
\hline 3 & 3 & -3 & 6 & -6 & 9 & -9 & 12 & -12 & 15 & -15 \\
\hline-3 & -3 & 3 & -6 & 6 & -9 & 9 & -12 & 12 & -15 & 15 \\
\hline 4 & 4 & -4 & 8 & -8 & 12 & -12 & 16 & -16 & 20 & -20 \\
\hline-4 & -4 & 4 & -8 & 8 & -12 & 12 & -16 & 16 & -20 & 20 \\
\hline 5 & 5 & -5 & 10 & -10 & 15 & -15 & 20 & -20 & 25 & -25 \\
\hline-5 & -5 & 5 & -10 & 10 & -15 & 15 & -20 & 20 & -25 & 25 \\
\hline
\end{tabular}

что, конечно, облегчает ее запоминание. В статье он приводит пример умножения чисел

\section{5 и 389175836438}

после преобразования их в соответствующие числа с отрицательными цифрами

$$
1 \overline{1} \overline{4} 1 \overline{4} \overline{3} 3 \overline{1} 40 \overline{1} \overline{3} 15 \text { и } 4 \overline{1} \overline{1} 2 \overline{2} \overline{4} \overline{2} 4 \overline{4} 44 \overline{2}
$$

и получает результат, равный

\section{0 (!).}

\section{Отрицательные цифры Коши}

Более чем через 100 лет после статьи Колсона, в 1840 году, использовать отрицательные цифры также предложил (с той же целью) французский математик Огюстен Луи Коши [2], скорее всего, не знакомый с работой Колсона. Правда, он не привел примеры расчетов, но обратил внимание на то, что квадраты чисел 11, 12 и 13 (равные, соответственно, 121, 144 и 169) аналогичны квадратам чисел с отрицательными цифрами: $(1 \overline{1})^{2}=1 \overline{2} 1,(1 \overline{2})^{2}=1 \overline{4} 4$ и $(1 \overline{3})^{2}=1 \overline{6} 9$ и что периодические дроби с шестизначным периодом

$$
\begin{aligned}
& 1 / 7=0,142857142857 \ldots \\
& 1 / 13=0,076923076923 \ldots
\end{aligned}
$$

при использовании в них отрицательных цифр становятся периодическими с, мож- но сказать, «трехзначным периодом»:

$$
\begin{aligned}
& 0,143 \overline{1} \overline{4} \overline{3} 143 \overline{1} \overline{4} \overline{3} \ldots, \\
& 0,1 \overline{2} \overline{3} \overline{1} 231 \overline{2} \overline{3} \overline{1} 231 \ldots
\end{aligned}
$$

Интересно, что Коши обнаружил также такие «аналогичные» значения:

$$
1013^{2}=1026169 \text { и }(10 \overline{1} \overline{3})^{2}=10 \overline{2} \overline{6} 169 \text {, }
$$
$1006^{3}=1018108216$ и

$$
(100 \overline{6})^{3}=10 \overline{1} \overline{8} 108 \overline{2} \overline{1} \overline{6} .
$$

\section{Отрицательные цифры Селлинга}

В 1887 году немецкий математик Эдуард Селлинг в своей работе [3], описывающей изобретенную им вычислительную машину, рекомендовал использовать отрицательные цифры 1, 2, 3, 4 и 5, записывая их вверх тормашками: I, Z, \&, Ғ, S. Это позволяло отказаться от цифр $9,8,7$ и 6 , что существенно упрощало расчеты. Так, «обычное» число 1046207753300 он приводит в виде: 105§217Zऽ3300. Причем, по мнению Селлинга, использование двух вариантов цифры 5 является целесообразным.

Ученый также предложил варианты написания таких цифр буквами (и, соответственно, их произношения):

1) с записью немецких слов eins (один), zwei (два), drei (три), vier (четыре) и fünf (пять) в виде snie, jes, jerd, reff и niff (некоторые слова записаны «задом наперед»); такой вариант он посчитал не очень подходящим;

2) с приставкой miss, означающей отрицание чего-либо: missein («неодин»), mizwei («недва»), midrei («нетри») и т.п. (в ряде случаев приставка сокращается); Селлинг приводит пример произношения числа $5 z z$ - «ünf hundert mizwanzig mizwei» ( «пятьсот недвадцать недва»);

$3)$ с приставкой $a b$, означающей отсутствие чего-либо: abein, abzwei, abdrei и т.д. По мнению ученого, такой вариант лучше других подходит к немецкому языку и другим языкам, использующим латинские буквы.

Интересно, что в 1925 году американский математик Джон Баллантайн предложил использовать перевернутую обычную единицу (I) для записи так называемого 
«дополнительного кода отрицательных чисел», применявшегося в вычислительных машинах. По его мнению, такая запись будет более привычной для человека, чем цифра 9 в начале кода. Конечно, это утверждение относилось к вычислительным машинам, работавшим с десятичными числами (а не с двоичными, как современные компьютеры). Заметим, что на популярном ресурсе «Википедия» в качестве недостатка представления отрицательного числа в виде дополнительного кода указывается то, что такой код «... визуально не читается по обычным правилам, для его восприятия нужен особый навык», что совпадает, по сути, с утверждением Баллантайна.

\section{Задача об уравновешивании весов}

А для каких практических целей могут быть использованы отрицательные цифры?

В качестве примера можно привести такую задачу: «Известна масса груза $K$ г. Какой минимальный набор гирь необходимо использовать для того, чтобы уравновесить чашечные весы, и как разместить гири для этого, если их можно класть на обе чашки весов?»

Решение этой задачи основано на следующей особенности: «Любое целое положительное число может быть получено в виде суммы или / и разности чисел, являющихся степенью тройки». Вот несколько примеров:

$$
\begin{aligned}
& 1=1 \\
& 2=3-1 \\
& 4=3+1 \\
& 5=9-3-1 \\
& 6=9-3 \\
& 7=9-3+1 \\
& 21=27-9+3 \\
& 89=81+9-1
\end{aligned}
$$

Это значит, что минимальный набор гирь для взвешивания груза, например, от 1 до 100 г такой: 1, 3, 9, 27 и 81 г. Так как на чашечных весах гири можно размещать на обеих чашках - и на свободной, и вместе с грузом, то для уравновешивания на них груза массой $K$ г нужно на свободную

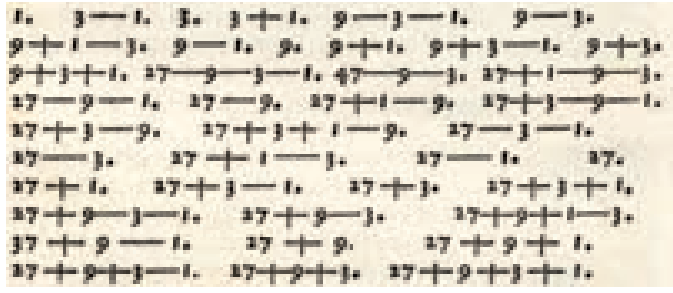

Представления чисел от 1 до 27 в виде суммы или разности степеней тройки из книги "Arithmetica Integra» немецкого монаха Михаэля Штифелиуса (1487-1567), выпущенной в 1545 году

чашку весов положить гири массой, значения которых для данного груза в правой части соответствующего равенства записаны со знаком «+», а на чашку вместе с грузом - гири массой со значениями со знаком «-» (если такие значения имеются):

\begin{tabular}{|c|c|c|}
\hline $\begin{array}{c}\text { Мacса груза } \\
K, \Gamma\end{array}$ & $\begin{array}{c}\text { На чашке весов } \\
\text { с грузом }\end{array}$ & $\begin{array}{c}\text { На другой } \\
\text { чашке }\end{array}$ \\
\hline 1 & & 1 \\
\hline 2 & 1 & 3 \\
\hline 4 & & 1 и 3 \\
\hline 5 & 1 и 3 & 9 \\
\hline 6 & 3 & 9 \\
\hline 7 & 3 & 1 и 9 \\
\hline 21 & 9 & 3 и 27 \\
\hline 89 & 1 & 9 и 81 \\
\hline
\end{tabular}

Но при чем тут отрицательные цифры? Давайте разбираться.

\section{Троичная уравновешенная система счисления}

Как известно, любое числовое значение можно записать не только в используемой нами десятичной системе счисления, но и в системе с другим основанием. Например, число $K$ в троичной системе можно представить как

$$
K=\left(a_{n} a_{n-1} \ldots a_{1} a_{0}\right)_{3},
$$

или (в так называемой «развернутой форме записи»)

$$
K=a_{n} \cdot 3^{n}+a_{n-1} \cdot 3^{n-1}+\ldots+a_{1} \cdot 3+a_{0},
$$

где цифры $a_{0}, a_{1}, \ldots, a_{n}$ могут принимать значения 0, 1 или 2.

Можно доказать, что $2 \cdot 3^{m}=3^{m+1}-3^{m}$. Введем «отрицательную цифру» 1 и обо- 
значим ее $\overline{1}$. Тогда последнее равенство можно записать в виде $2 \cdot 3^{m}=3^{m+1}+\overline{1} \cdot 3^{m}$. А это означает, что любое целое число $K$ можно изобразить в троичной системе счисления с помощью цифр 0, 1 и $\overline{1}$ (заменив в его развернутой записи все цифры 2 на соответствующую разность):

$$
K=b_{m} \cdot 3^{m}+b_{m-1} \cdot 3^{m-1}+\ldots+b_{1} \cdot 3+b_{0},
$$

где каждый из коэффициентов $b_{m}, b_{m-1}, \ldots$ $\ldots, b_{0}$ может быть равным 0, 1 или $\overline{1}$. Такой способ записи чисел называется «троичной уравновешенной системой счисления». ${ }^{2}$ Так вот, если мы знаем, как записывается масса груза $K$ в этой системе, то, чтобы уравновесить его на весах, нужно положить его на первую чашку весов, а гирю в 1 г поставить на вторую чашку, если $b_{0}=1$, и на первую чашку, если $b_{0}=\overline{1}$ (если $b_{0}=0$, то гиря в 1 г вообще не используется); далее, гиря в 3 г ставится на вторую чашку, если $b_{1}=1$, и на первую, если $b_{1}=\overline{1}$, и т.д. Легко понять, что, расставив гири по такому принципу, мы уравновесим груз.

Прежде чем приводить пример, покажем, как преобразовать обычную троичную запись в запись в уравновешенной троичной системе. Для этого нужно для каждой двойки выполнить следующие действия:

1) заменить ее на цифру $\overline{1}$;

2) в соседнем слева разряде добавить 1 (если в этом разряде в результате получается 2, то для него указанные действия повторяются).

Например, число 130, которое обычным образом записывается в троичной системе как 11211, во втором варианте будет иметь вид

$$
1 \overline{1} \overline{1} 111\left(3^{5}-3^{4}-3^{3}-3^{2}+3^{1}+3^{0}=130\right) \text {. }
$$

Таким образом, чтобы уравновесить груз в 130 граммов, положенный на одну из

2 Впервые такую систему описал французский инженер Леон Лаланн в 1840 году. Он привел несколько первых натуральных чисел в этой системе:

$1,1 \overline{1}, 10,11,1 \overline{1} \overline{1}, 110,1 \overline{1} 1,10 \overline{1}, 100,101,11 \overline{1}, \ldots$ чашек весов, нужно на ту же чашку положить гири в 81, 27 и 9 граммов, а на противоположную - гири в 243, 3 и 1 грамм. В решении задачи нам помогла уравновешенная троичная система счисления. ${ }^{3}$

В заключение заметим, что «самый реальный» пример использования отрицательных цифр - это электронно-вычислительные машины «Сетунь» 4 и «Сетунь 70», разработанные, соответственно, в 1960 и 1970 году в Московском государственном университете имени М.В.Ломоносова под руководством Николая Петровича Брусенцова (1925-2014). Их устройство и работа были основаны на уравновешенной троичной системе счисления с цифрами -1, 0 и 1. [5]

\section{Еще об уравновешенной троичной системы счисления}

Дональд Кнут, автор всемирно известной серии книг, посвященной основным алгоритмам и методам вычислительной математики, в книге [6, стр. 230-231] приводит другой метод перевода. ${ }^{5}$ Он состоит в следующем.

1. К троичному числу прибавляется число из одних единиц. Например, для $130_{10}=11211_{3}$ :

$$
\begin{array}{r}
11211 \\
+\begin{array}{r}
11111 \\
\hline 100022
\end{array}
\end{array}
$$

2. Из полученной суммы вычитается (поразрядно) число из одних единиц, использовавшееся при сложении (каждая цифра уменьшаемого уменьшается на 1):

$$
\begin{aligned}
& 100022 \\
& \frac{11111}{1 \overline{1} \overline{1} \overline{1} 11}
\end{aligned}
$$

\footnotetext{
3 Впервые на связь задачи о наборе гирь и уравновешенной троичной системы указал Люка в книге «Математические развлечения» [4]. Правда, ученый не описал, как решить задачу для конкретного значения веса груза.

4 Сетунь - название речки, протекающей неподалеку от МГУ.

5 Ученый называет уравновешенную троичную систему «быть может, самой изящной из всех систем счисления».
} 
Результат совпадает с полученным в нашей статье.

\section{Упражнения}

1. Запишите в уравновешенной системе троичные числа:

a) 210 ;

б) 1202

2. Запишите в уравновешенной троичной системе десятичные числа:

a) 17 ;

б) 53 .

\section{Свойства уравновешенной троичной системы счисления}

У равновешенная троичная система счисления обладает многими весьма привлекательными свойствами.

Во-первых, она дает возможность единообразно выражать как положительные, так и отрицательные числа (как отмечалось выше, при использовании двоичной системы отрицательные числа в компьютере представляются в так называемом «дополнительном коде»). В ней знак числа определяется первым символом в записи числа в этой системе: если он равен 1, то число положительное, а если $-1(\overline{1})$, то отрицательное.

Очень просто перейти к противоположному числу, заменив 1 на $-1(\overline{1})$ и наоборот. В самом деле:

$$
-8_{10}=\overline{1}_{01} ; \quad 8_{10}=10 \overline{1}_{3} .
$$

Это значит, что сравнивать любые два числа можно как в десятичной системе, сравнивая цифры слева направо.

Для округления вещественного троичного числа до ближайшего целого достаточно отбросить его дробную часть. Это свойство также достаточно очевидно, поскольку самая большая возможная положительная дробная часть $0,111 \ldots$ представляет сумму $1 / 3+1 / 9+1 / 27+\ldots$, которая всегда меньше $1 / 2$.

Арифметические операции в троичной симметричной системе практически не сложнее двоичных, а если учесть, что в случае чисел со знаком двоичная арифметика использует искусственные коды, то окажется, что троичная даже проще. Операция сложения всякой цифры с нулем дает в результате эту же цифру. Сложение $+1 \mathrm{c}-1$ дает ноль. И только сумма двух +1 или двух -1 формируется путем переноса в следующий разряд цифры того же знака, что и слагаемые, и установки в текущем разряде цифры противоположного знака. Полностью таблица сложения, которой будем пользоваться для обычного сложения чисел «столбиком», имеет вид

\begin{tabular}{|c|c|c|c|}
\hline+ & $\overline{1}$ & 0 & 1 \\
\hline$\overline{1}$ & $\overline{1} 1$ & $\overline{1}$ & 0 \\
\hline 0 & $\overline{1}$ & 0 & 1 \\
\hline 1 & 0 & 1 & $1 \overline{1}$ \\
\hline
\end{tabular}

Примечание. Запись $1 \overline{1}$ означает, что результат равен $\overline{1}$ и еще 1 переносится в следующий разряд, запись $\overline{1} 1$ - что результат равен 1 и еще $\overline{1}$ переносится в следующий разряд.

Сложим в уравновешенной троичной системе числа 412 и 181:

$$
\begin{aligned}
412_{10}= & 120021_{3}=1 \overline{1} \overline{1} 01 \overline{1} 1_{3} \\
181_{10}= & 20201_{3}=1 \overline{1} 1 \overline{1} 01_{3} \\
& +\frac{1 \overline{1} \overline{1} 01 \overline{1} 1}{1 \overline{1} 1 \overline{1} 01} \\
& +\overline{1} 1100 \overline{1}
\end{aligned}
$$

Легко проверить, что $1 \overline{1} 1100 \overline{1}_{3}=593_{10}$.

Упражнение 3. Найдите в уравновешенной троичной системе сумму чисел:

a) $10 \overline{1} 1 \overline{1}$ и $1 \overline{1} 0 \overline{1}$;

б) $1 \overline{1} \overline{1} 011$ и $1 \overline{1} 011$

Результаты представьте в десятичной системе.

Столь же просто производится вычитание: для этого достаточно изменить знак вычитаемого на противоположный и сложить число с уменьшаемым. Конечно, можно проводить и «непосредственное» вычитание. Для этого удобно составить таблицу вычитания. Обсудим ее, поскольку при составлении такой таблицы получается ряд интересных результатов.

Для следующих случаев ничего необычного нет:

1) $0-0=0$;

2) $1-0=1$

3) $1-1=0$;

4) $0-\overline{1}=1$ (это следует из правил алгебры и из таблицы сложения, приведенной выше);

5) $\overline{1}-\overline{1}=0$ (по тем же причинам). 
А теперь - то самое, интересное.

Сколько будет 1- 1 ? Конечно, $1 \overline{1}$ (по правилам алгебры $1-(-1)=2=1 \overline{1})$. Но возникает вопрос: что происходит в соседнем слева разряде при вычитании многозначных чисел - единица заимствуется или добавляется? Для ответа рассмотрим пример:

$$
\begin{gathered}
10_{10}-2_{10}=101_{3}-1 \overline{1}_{3} \\
-\frac{10 \frac{1}{1}}{10 \overline{1}}
\end{gathered}
$$

(Результат равен 8 . .)

Анализ второго справа разряда показывает, что $\overline{1}$, «перешедшая» из крайнего правого разряда, складьвается с цифрами 0 и 1 !

Аналогично, для варианта $\overline{1}-1=\overline{1} 1$ цифра $\overline{1}$ переносится в следующий разряд, где также добавляется.

\section{Упражнения}

4. Составьте полную таблицу вычитания в уравновешенной троичной системе, в которой учитывается возможный перенос из разряда справа:

\begin{tabular}{|c|c|c|c|c|c|c|c|c|c|}
\hline \multirow{3}{*}{ Вычитаемое } & \multicolumn{7}{|c|}{ Перенос из разряда справа } \\
\cline { 2 - 10 } & \multicolumn{6}{|c|}{0} & \multicolumn{7}{|c|}{1} & \multicolumn{3}{|c|}{$\overline{1}$} \\
\hline & \multicolumn{7}{|c|}{ Уменьшаемое } \\
\hline & $\overline{1}$ & 0 & 1 & $\overline{1}$ & 0 & 1 & $\overline{1}$ & 0 & 1 \\
\hline$\overline{1}$ & & & & & & & & & \\
\hline 0 & & & & & & & & & \\
\hline 1 & & & & & & & & & \\
\hline
\end{tabular}

5. Найдите разность в уравновешенной троичной системе (используя составленную таблицу или заменив вычитание сложением):

a) $10 \overline{1} 1-\overline{1} \overline{1} 1$;

б) $11 \overline{1} \overline{1} 0-1 \overline{1} 11$.

Результаты представьте в десятичной системе.

Таблица умножения - совсем простая: умножение на ноль дает ноль, умножение на 1 повторяет множимое, умножение на -1 инвертирует множимое (заменяет 1 на -1 , а -1 на 1 , т.е. изменяет знак числа):

$$
\begin{array}{|c|c|c|}
\hline \times & \overline{1} & 1 \\
\hline \overline{1} & 1 & \overline{1} \\
\hline 1 & \overline{1} & 1 \\
\hline
\end{array}
$$

Умножение многозначных чисел сводится к простым операциям изменения знака (при необходимости) и сложения. Умножим, например, десятичные числа 5 и 12, записанные в уравновешенной троичной системе:

$$
\begin{gathered}
5_{10}=1 \overline{1} \overline{1}_{3} \\
12_{10}=110_{3} \\
\times 1 \overline{1} \overline{1} \\
\frac{110}{1 \overline{1} \overline{1}} \\
\frac{1 \frac{1}{1} \frac{1}{1}}{1 \overline{1} 1 \overline{1} 0}
\end{gathered}
$$

или

$$
\begin{gathered}
\times 110 \\
11 \overline{1} \\
\hline 110 \\
110 \\
110 \\
\hline 1 \overline{11} \overline{10}
\end{gathered}
$$

Переведя троичное число $1 \overline{1} 1 \overline{1} 0$ в десятичную систему, получим 60 (т.е. результат правильный).

\section{Упражнения}

6. Определите произведение чисел 20 и -19 , записанных в уравновешенной троичной системе. Результат представьте в десятичной системе.

7. Опишите также правила перевода числа, представленного в уравновешенной троичной системе, в «обычную» троичную систему

а) применительно к положительному числу;

б) применительно к отрицательному числу.

\section{Литература}

1. J.Colson. A Short Account of NegativoAffirmative Arithmetick. https:// royalsocietypublishing.org/doi/10.1098/ rstl.1726.0032

2. A.-L.Cauchy. Oeuvres. Serie I. Tome V.Paris, 1885. https://books.google.ru/books?id= jE4NAAAAYAAJ

3. E.Selling. Eine neue Rechenmaschine. Berlin, 1887.

4. E.Lucas. Récréations mathématiques. https:// archive.org/stream/rcrationsmathma00lucagoog\# page/n26/mode/1 up

5. https://ru.wikipedia.org/wiki/Сетунь_ (компьютер)

6. Д.Кнут. Искусство программирования Том 2. 3-е издание. - М.: Диалектика-Вильямс, 2001. 